Check for updates

Cite this: Soft Matter, 2020, 16,8512

DOI: $10.1039 / \mathrm{dOsm} 90174 \mathrm{a}$

rsc.li/soft-matter-journal

\section{Correction: Transient coarsening and the motility of optically heated Janus colloids in a binary liquid mixture}

\author{
Juan Ruben Gomez-Solano, ${ }^{a}$ Sutapa Roy, ${ }^{\text {bc }}$ Takeaki Araki, ${ }^{d}$ S. Dietrich ${ }^{\text {bc }}$ and \\ Anna Maciotek*bce
}

Correction for 'Transient coarsening and the motility of optically heated Janus colloids in a binary liquid mixture' by Juan Ruben Gomez-Solano et al., Soft Matter, 2020, DOI: 10.1039/d0sm00964d.

There is a missing Appendix in the original article. Additionally, the Appendix titled 'Diffusive model' in the original article is incorrectly labelled as Appendix A; this should be Appendix B. Appendix A is as below:

\title{
A Determination of the local temperature
}

In order to determine the local temperature of the gold-coated side of the particle under green illumination, we take advantage of the visibility of the fluid droplet formed around the particle surface when heated above $T_{\mathrm{c}}=31.9{ }^{\circ} \mathrm{C}$. First, we set the temperature of both the binary mixture and the Janus particle to $T_{0}=28^{\circ} \mathrm{C}$ by means of a thermostat in contact with the sample cell. This is the temperature at which all experiments described in the present paper are performed. Next, we apply green illumination to the particle (intensity $I=2 \mu \mathrm{W} \mu \mathrm{m}^{-2}$ ), which is sufficient to observe the coarsening patterns investigated here, until the final steady-state droplet is formed, as shown in Fig. 14(a). This value of $I$ corresponds to the intensity of the incident green light reported in the main text. By keeping $T_{0}$ constant, we slowly decrease the light intensity $I$. In this way, the absolute temperature of the golden cap, $T_{0}+\Delta T$, also decreases, thereby decreasing the size of the resulting droplet, see Fig. 14(b) and (c). On the other hand, the unknown temperature rise of the cap (with respect to $T_{0}$ ) due to light absorption depends linearly on I, i.e., $\Delta T=k I$, where $k$ is a constant which depends on the particle size and the thickness of the gold cap. The value of $I$ at which the droplet completely disappears corresponds to a local temperature of the golden cap at the critical value, i.e., $T_{0}+\Delta T=T_{\mathrm{c}}$. We find that this occurs at $I=1 \mu \mathrm{W} \mu \mathrm{m}^{-2}$, as shown in Fig. 14(d), from which we infer that $k=3.9{ }^{\circ} \mathrm{C} \mu \mathrm{W}^{-1} \mu \mathrm{m}^{2}$, thus leading to a local temperature rise of $\Delta T=7.8^{\circ} \mathrm{C}$ at $I=2 \mu \mathrm{W} \mu \mathrm{m}^{-2}$.
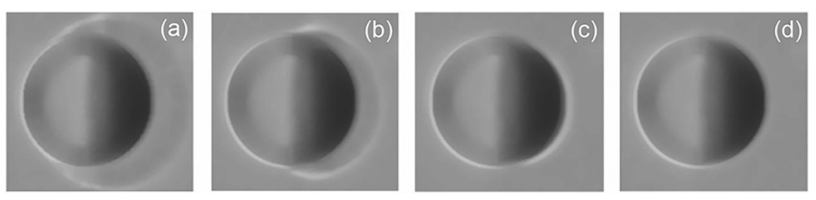

Fig. 14 Snapshots of the steady-state concentration profiles of the $\mathrm{PnP}$-water mixture around a spherical Janus colloid (hydrophilic gold cap) under various illumination intensities: (a) $I=2.0 \mu \mathrm{W} \mu \mathrm{m}^{-2}$, b) $I=1.7 \mu \mathrm{W} \mu \mathrm{m}^{-2}$, c) $I=1.3 \mu \mathrm{W}_{\mu} \mathrm{m}^{-2}$, and d) $I=1.0 \mu \mathrm{W} \mu \mathrm{m}^{-2}$. In all cases, the sample cell is kept at $T_{0}=28{ }^{\circ} \mathrm{C}$ by means of a thermostat.

Additionally, the authors note the description for some of the symbols and their colours are incorrect within Section 3.1.4. Any expression of (blue squares in Fig. 4(a)) should be (black squares in Fig. 4(a)), any expression of (black squares in Fig. 4(b)) should be (black diamonds in Fig. 4(b)) and any expression of (red squares in Fig. 7(b)) should be (black squares in Fig. 7(b)).

\footnotetext{
${ }^{a}$ Instituto de Fisica, Universidad Nacional Autonoma de México, Apdo. Postal 20-364, 01000, Ciudad de México, Mexico

${ }^{b}$ Max-Planck-Institut für Intelligente Systeme, Heisenbergstr. 3, 70569 Stuttgart, Germany

${ }^{c}$ IV. Institut für Theoretische Physik, Universität Stuttgart, Pfaffenwaldring 57, 70569 Stuttgart, Germany

${ }^{d}$ Department of Physics, Kyoto University, Kyoto 606-8502, Japan

${ }^{e}$ Institute of Physical Chemistry, Polish Academy of Sciences, Kasprzaka 44/52, PL-01-224 Warsaw, Poland. E-mail: amaciolek@ichf.edu.pl
} 
Furthermore, in Section 3.2.2, in the last paragraph, $T=-1$ should be $\mathscr{T}_{1}=-1$.

The Royal Society of Chemistry apologises for these errors and any consequent inconvenience to authors and readers. 